\title{
The SNLS-VLT Type Ia Spectrum Evolution with Redshift: a Demographic Effect?
}

\author{
Christophe Balland ${ }^{1,2}$ and Martine Mouchet $^{3}$ \\ ${ }^{1}$ LPNHE, Universités Paris 6 \& 7, \\ 4 Place Jussieu, 75252 Paris Cedex 05, France \\ email: balland@lpnhe.in2p3.fr \\ ${ }^{2}$ Université Paris 11, Orsay, F-91405, France \\ ${ }^{3}$ APC, Université Paris Diderot - Paris 7, \\ 10, Rue Alice Domon et Léonie Duquet, 75205 Paris Cedex 13, France \\ email: martine.mouchet@obspm.fr
}

\begin{abstract}
We build composite spectra at $z \leqslant 0.5$ and $z>0.5$ from the 3 year SuperNova Legacy Survey (SNLS) VLT spectral sample and study their differences around maximum light. We use 93 spectra near maximum of confirmed SNe Ia observed at the VLT between 2003 and 2006 as part of the 3 first years of operation of the SNLS. We find differences in the absorption depth of some intermediate mass elements (Ca II , Si II). Average stretches of observed distributions are $0.975 \pm 0.016$ and $0.983 \pm 0.014$ for the $z \leqslant 0.5$ and $z>0.5$ sample respectively. We use the A + B model of Scannapieco \& Bildsten (2005) to interpret these results in terms of a possible demographic evolution.
\end{abstract}

Keywords. stars: evolution, supernovae — techniques: spectroscopy

\section{Composite Spectra}

The individual Type Ia supernovae (SNe Ia) spectra used to build the composite spectra under investigation in this study have been obtained at the VLT between 2003 and 2006 and are presented in Balland et al. (2009). A dedicated reduction and identification pipeline has been set up to obtain an optimized homogeneous sample (Baumont et al. 2008). Host subtraction is performed using a photometric model of the host built from deep MEGACAM reference stacked images. SNe Ia spectra are then selected according to their effective phase $\phi$, defined as the number of days elapsed in the rest-frame between the B-band maximum light and the date the spectrum was acquired, divided by the stretch $s$. A subset of 93 spectra with effective phases between -4 and 4 days are used to study differences around maximum light. Twenty-five are at $z \leqslant 0.5$ and 68 at $z>$ 0.5. Rest-frame spectra are normalized around $4500 \AA$ and averaged. A weighted error is computed at each wavelength from the individual error spectra considered in the averaging process. Figure 1 shows the resulting composite spectra at maximum light for the two redshift bins (see Balland et al. 2009 for details). Table ?? lists the redshift and stretch average values of the SNe Ia used in each redshift bin. Differences are seen in the absorption depths of Si II at $4100 \AA$ and Ca II around $3600 \AA$. As shown in Balland et al. (2009), these are not due to a different phase sampling of spectra in the two redshift bins. More likely, they result from higher redshift SNe Ia in our sample being on average more luminous and hotter than their low redshift counterparts, a fact that has 


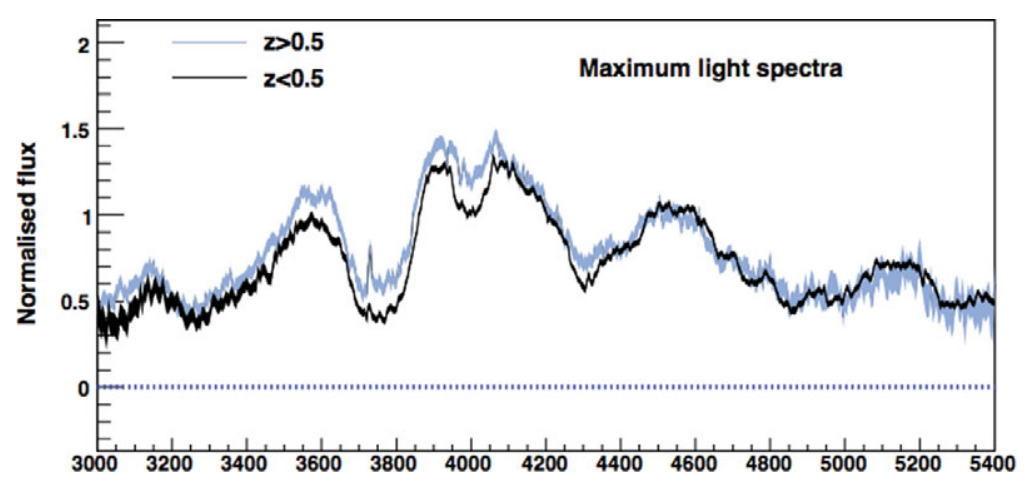

Figure 1. Composite spectra of SNe Ia around maximum at $z<0.5$ and $z>0.5$.

Table 1. Average stretch and redsfhit of the SNe Ia in the two redshift bins considered in this study.

\begin{tabular}{c|c|c} 
& $z \leqslant 0.5$ & $z>0.5$ \\
\hline \# SN Ia & 25 & 68 \\
$<z>$ & $0.361 \pm 0.021$ & $0.698 \pm 0.018$ \\
$<s>$ & $0.975 \pm 0.016$ & $0.983 \pm 0.014$ \\
\hline
\end{tabular}

already been noticed by several authors (e.g., Sullivan et al. 2009). We investigate below whether this fact can be explained by a demographic evolution of SNe Ia populations with redshift.

\section{Testing the Demographic Evolution Hypothesis}

Following Howell et al. (2007), we use the A+B model of Scannapieco \& Bildsten (2005) to identify a prompt and a delayed component in our spectral sample. All SNe Ia from passive hosts (based on their specific star formation rate) are assigned to the A (delayed) component. To obtain the B (prompt) component, we scale by mass the A distribution from passive hosts and we subtract it from the distribution of SNe Ia in star forming galaxies. The average stretch is $0.960 \pm 0.009$ for the A component, and is $1.018 \pm 0.003$ for the $\mathrm{B}$ component. This is consistent with finding the low-stretch, fainter SNe Ia in passive hosts. To model the stretch distributions of the $z \leqslant 0.5$ and $z>0.5$ sub-samples, we perform Gaussian fits to the A and B distributions and scale them to the predicted rates of A and B SNe Ia at the average redshift of the two redshift bins, following Sullivan et al. (2006). Figure 1 shows the model predictions for the $\mathrm{A}, \mathrm{B}$ and $\mathrm{A}+\mathrm{B}$ components (solid curves), compared to the observed stretch distributions (histograms) at low and high redshift. We find that the observed distributions are reproduced by the $\mathrm{A}+\mathrm{B}$ model, with $<s>_{z \leqslant 0.5}^{A+B}=0.980 \pm 0.016$ and $<s>_{z>0.5}^{A+B}=0.989 \pm 0.010$. The model predicts a mild demographic evolution, the ratio $\mathrm{A} / \mathrm{A}+\mathrm{B}$ ratio evolving from 0.65 at $z=0.36$ to 0.5 at $z=0.70$. This is in agreement with the findings of Howell et al. (2007), given the smaller lever arm in redshift of the present study. 

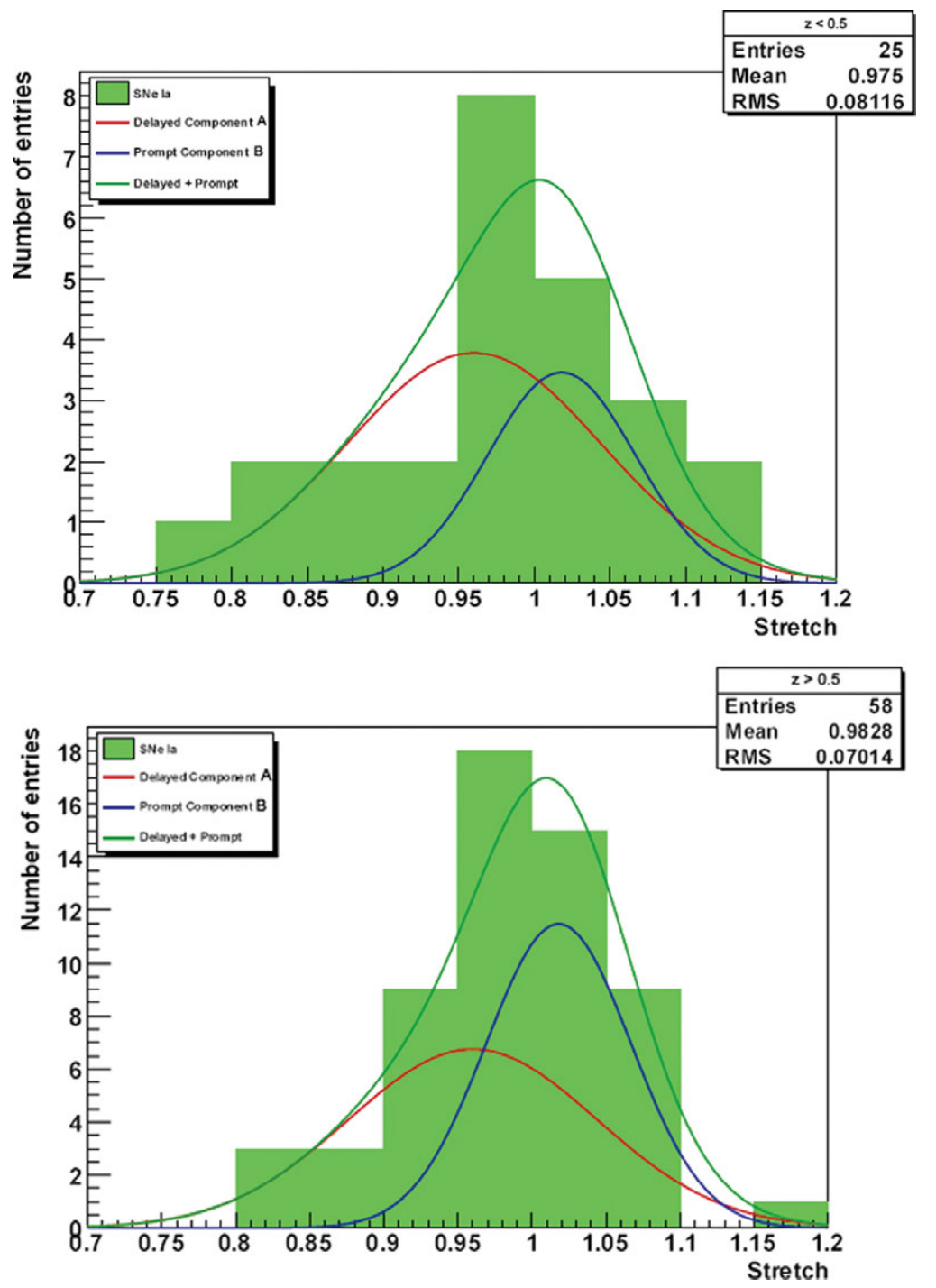

Figure 2. Stretch distributions of the $z \leqslant 0.5$ and $z>0.5$ sub-samples. A+B model predictions are overlapped.

\section{References}

Balland C., Baumont S., Basa S., et al. 2009, A\&A, 507, 85

Baumont S., Balland C., Astier P., et al. 2008, A\& A, 491, 567

Howell D. A., Sullivan M., Conley A. \& Carlberg, R. 2007, ApJ, 667, 37

Scannapieco E. \& Bildsten L. 2005, ApJ, 629, 85

Sullivan M., Le Borgne D., \& Pritchet C. J. 2006, ApJ, 648, 868

Sullivan M., Ellis R. S., Howell D. A., et al. 2009, ApJL, 693, L76 